\title{
Face Recognition Based on Statistical Texture Features
}

\author{
Lama Ibrahim \\ Department of Software and Information Systems \\ Tishreen University \\ Latakia, Syria \\ lamaakramibrahim@tishreen.edu.sy
}

\author{
Nasser Nasser \\ Department of Software and Information Systems \\ Tishreen University \\ Latakia, Syria \\ n.nasser@tishreen.edu.sy
}

\author{
Majd Ali \\ Department of computers and automatic control \\ engineering \\ Tishreen University \\ Latakia, Syria \\ majed.ali.1976@tishreen.edu.sy
}

\begin{abstract}
Facial recognition has attracted the attention of researchers and has been one of the most prominent topics in the fields of image processing and pattern recognition since 1990. This resulted in a very large number of recognition methods and techniques with the aim of increasing the accuracy and robustness of existing systems. Many techniques have been developed to address the challenges and reliable recognition systems have been reached but require considerable processing time, suffer from high memory consumption and are relatively complex. The focus of this paper is on extracting subset of descriptors (less correlated and less calculations) from the co-occurrence matrix with the goal of enhancing the performance of Haralick's descriptors. Improvements are achieved by adding the image preprocessing and selecting the proper method according to the database problem and by extracting features from image local regions.
\end{abstract}

Keywords-face recognition;texture, Haralick features; GLRLM; illumination pre-processing; local approach; SVM.

\section{INTRODUCTION}

Traditional authentication systems are classified into knowledge-based systems and token-based systems. Knowledge-based systems use usernames and passwords while token-based systems use tokens (identification card, smart card, passport, keys ..). The main problems of these systems are the possibility of forgetting passwords, forgery, loss of identification cards, card damage. In recent years, biometric authentication systems have emerged as a promising option for identifying individuals. These techniques identify individuals by analyzing their physiological and behavioral characteristics. Its advantage is that it is not forgotten, stolen, or forged and needs the presence of the person during the authentication process.

\footnotetext{
${ }^{1}$ Copyright $@ 2020$ by ESS Journal
}

Compared with other biometrics, facial recognition is one of the least intrusive and fastest biometric. So facial recognition systems can be used in surveillance systems, take pictures of people faces when they enter a certain area unnoticed and they do not feel under surveillance. But this biometric system has many constraints resulting from many challenges. Facial recognition in a controlled environment has reached a saturation point but the problem remains open in an uncontrolled environment due to significant changes in illumination conditions, facial expressions, age, head orientation, occlusion (beard, glasses, ..). Many facial recognition systems are implemented to recognize human face 2D image, these systems were classified into three approaches [1]: Holistic approach, Local approach, Hybrid approach. The holistic approach was dominant in the 1990s, but it has a problem that it failed to address the uncontrolled facial changes. Local approach [2] is robust for illumination changes and facial expressions but sensitive to noise.

One important problem in face recognition is finding efficient descriptors for extracting the useful and discriminant features from facial images. The texture is a key component for human visual perception and is widely used in the field of computer vision. The texture analysis community worked to develop a lot of different descriptors for the appearance of images, but the problem of facial recognition was not related to this development as it was not verified from this point of view[3].

To build a robust and effective face recognition system, the problem of changing lighting is one of the main technical challenges facing system designers where a person's face looks very different. Lighting changes in the face image affect the classification error in a more important way be the effect caused by different people in the database[4].

Many different methods for analyzing texture have been developed that are based on the various ways texture can be 
characterized. In[5], a quick computational procedure was developed to derive a set of features based texture called Haralick features where the statistical nature of texture was taken into account. [6] also used texture features derived from gray-level run length matrix (GLRLM) to analysis satellite images by extracting the image information from gray level runs. But, these features used only runs length and its distribution so [7] suggested the use of gray level distribution due to the fact that the previous features aren't affected by the change of gray level value of a given run length distribution. So [8] introduced new GLRLM features based on the joint gray-level run-length distribution, it gave additional insight into image description and acted as effective features of texture-based image classification. Due to the existence of varying lighting and age, the local features were also introduced in face recognition systems. [3] presented a new and effective representation of face image based on the texture features Local Binary Pattern( LBP), which extracted locally from the image regions because they are more resistant to challenges of changing such as pose and lighting. Finding good descriptors for local facial regions is an open issue. Ideally, these descriptors should be easy to calculate and be able to distinguish between different individuals and be robust against changes that occur to the same person. [9] proposed a new, simple and straightforward method to face recognition based on features extracted from GLCM and focused also on the importance of quantization process. A new approach has been proposed [10] depending on local Haralick features rather than holistic for palmprint recognition. Local Haralick features are obtained by dividing the image into overlapping sub-regions. Then, GLCMs are calculated for each sub-region, from which local features are extracted and consider different values of the distance parameter that influences the GLCM.

Although there are many challenges addressing facial recognition, the problem of varying lighting is one of the biggest problems that hasn't been satisfactorily solved and a practical face recognition system has to deal with.

Most preprocessing methods perform almost perfectly in handling well- controlled lighting variations but they are still deficient in handling less-controlled illumination variations [11]. In [12] a comparative study of 12 representative illumination preprocessing methods were provided (HE, LT, GIC, DGD, LOG, SSR, GHP, SQI, LDCT, LTV, LN and TT). While [4] classified illumination preprocessing methods into two categories Relighting and Unlighting. They review and concise 64 state-of-the-art methods and indicated the most promising unlighting past state-of-the-art approaches such as single-scale Retinex, multi-scale Retinex, selfquotient image, discrete cosine transform. [13]demonstrated the effect of different pre-processing techniques on the performance of Haralick features such as Wavelet decomposition, local phase quantization, Weber law. The feature extraction algorithm performance has been improved, where Haralick features aren't only extracted from GLCM of the original image, but also from the image after filtering it with a Gaussian filter of different sizes.

The question is whether it is possible to apply effective features in the field of texture classification on the face and take advantage of the fact that these features are computationally easy, simple and fast. Then, improving the accuracy of their distinction through that local approach and adding pre-processing step.

\section{PROPOSED SYSTEM}

Face recognition can be used for two different tasks Identification or Verification. As illustrated in Figure 1, this study focuses on identification task which is one-to-many matching. We Divide face database into two sets: training $(70 \%)$ and testing $(30 \%)$. Perform 10 random splits and report the average result across 10 trials. Proposed system extracts training features to train classifier. When given new image from testing set, the trained classifier can decide the person's identity. We use two face databases ORL and YALE to evaluate the proposed system.

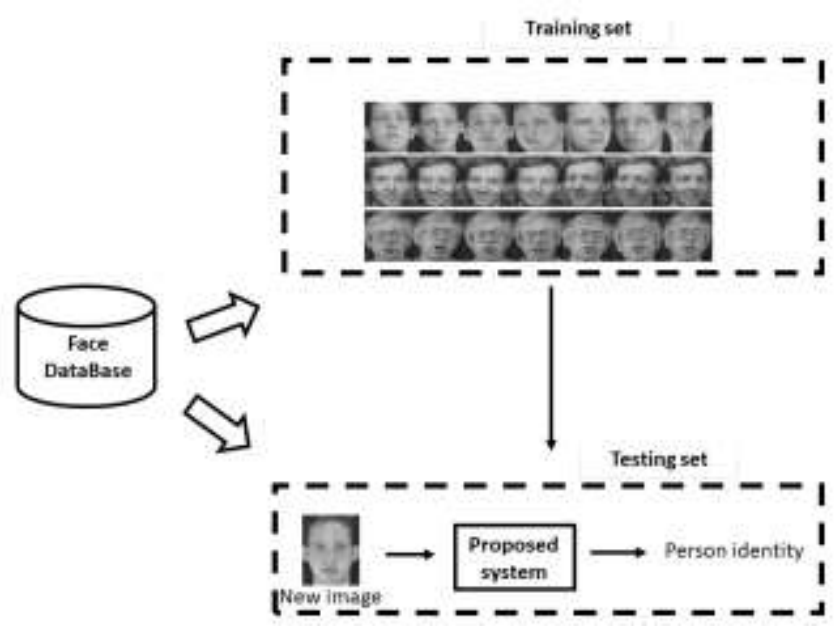

Figure 1: Identification scenario

Four basic steps are used to develop a robust face recognition system, as shown in Figure 2.

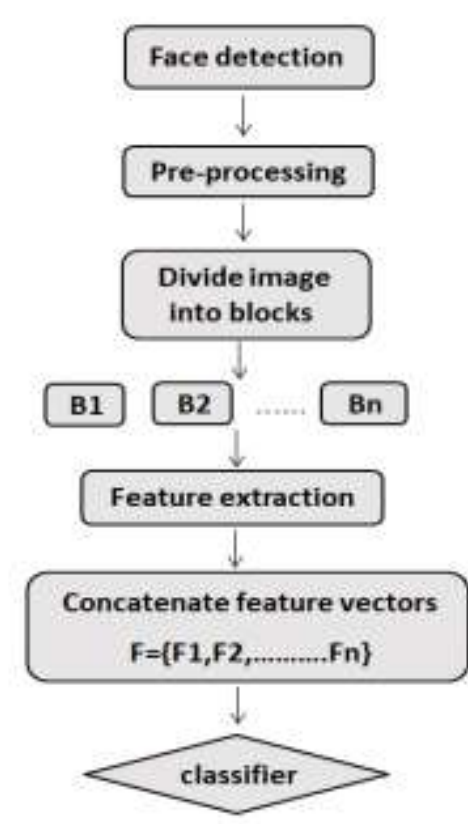

Figure 2: The proposed system

\section{A. Face detection :}

The purpose of this step is to detect and locate faces in images and, if any, returns the coordinates of a bounding 
box for each one of them. [14]oraganized the face detection algorithms into two categories rigid-template based algorithms and Deformable parts-based model. Rigidtemplate based algorithms are divided into Boosting algorithms and Deep Convolution Neural Network based algorithms.

An example of Boosting algorithms is the Viola Jones algorithm[15], the first algorithm that made face detection feasible in real-world applications and has the greatest impact in the 2000s and has so far been widely applied in digital cameras. It combines the concepts of Haar-like features, integral images, the AdaBoost algorithm and the Cascade classifier. Due to this Viola-Jones face detector is fast, robust and it got several implementations for different applications. Computer Vision System Toolbox ${ }^{\mathrm{TM}}$ supports different approaches to object detection in an image, including the Viola-Jones algorithm. In this paper, a practical implementation of a frontal view face detection algorithm based on Viola-Jones approach using Matlab cascade object detector is used [16].

As illustrated in Figure 3, we apply face detection step on YALE database imagaes to extract the face from the background. Then, resize all resulted images to $150 * 150$. Next, divide ORL images (size $112 * 92$ ) into $3 * 3$ local regions and YALE images into $3 * 4$ local regions as shown in Figure 4.

\section{B. Illumination pre-processing :}

Illumination Preprocessing methods are common due to their simplicity and the feasibility of applying them before
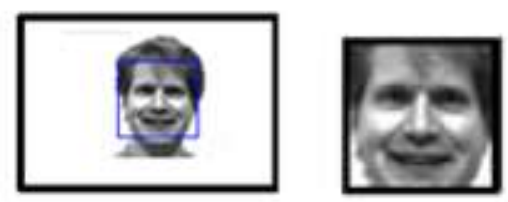

Figure 3: Bounding box found by a face detector to extract the face

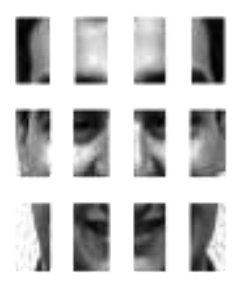

Figure 4: Face image divided into $3 * 4$ local regions

traditional face recognition methods. In addition, the output of this stage remains an image and therefore any feature descriptor can be applied after this stage. This step is necessary for the facial recognition system when it is done in an uncontrolled lighting conditions. Some mathematical models describing the reflectance of objects in computer graphics and computer vision were utilized to retrieve the facial image under various lighting conditions such as
Bidirectional Reflectance Distribution Function( BRDF) [17] and Lambertian model [18].

In [12], Illumination preprocessing approaches are analyzed from their principles and grouped into three categories:

1) Gray-level transformation : such as Histogram equalization(HE), logarithmic transform (LT), gamma correction(GC), these non-linear gray-level transformations have a role in the image enhancement, but can address the variation in lighting to some extent.

2) Gradient or edge extraction: such as gray-scale derivative(DGD), Laplacian of Gaussian(LoG).

3) Reflectance field estimation: face reflectance field is illumination-invariant and used to estimate the face reflectance field from a 2D face image such as single-scale Retinex (SSR), self-quotient image (SQI)[19], Logarithmic discrete cosine transform (LDCT) and TT[20].

TT, SQI and LDCT are found to be more effective for illumination-invariant face recognition in both wellcontrolled and less-controlled lighting conditions [12]. In this study, These methods are compared and choosing SQI (with three different scales for Gaussian filter) because it gives the best results on YALE database and applies GC (gamma $=2)$ to enhance ORL images (a homogeneous dark background ) as shown in Figure 5. Figure 6 shows how SQI compensated the lighting variation in the input image.

\section{Feature extraction :}

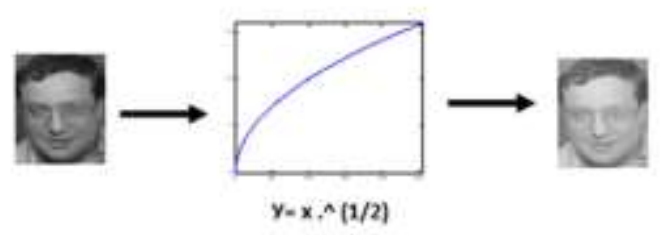

Figure 5: gamma correction is performed on a face image
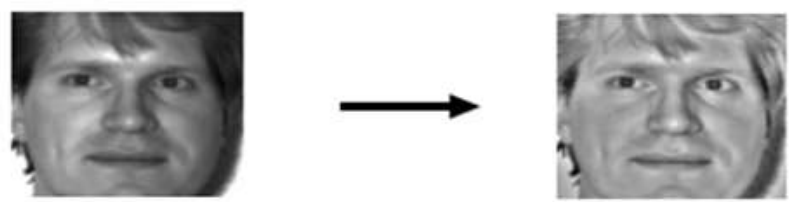

Figure 6: self quotient image is performed on a face image

Is the critical step in the face recognition system. The most popular and widely used statistical features in the field of texture analysis have been used.

1) Haralick features: GLCM is one of the earliest methods for analyzing texture, it proposed by Haralick [5] in 1979 for analyzing satellite images. The co-occurrence matrix counts the number of gray level transitions between two pixel values. The way pixel couples are determined depends on the two parameters, $d$ and $h$. A value of $d=1$ and $\mathrm{h}=0$, for example, would produce pixel couples that are adjacent to each other on the same row. A descriptor is obtained by concatenating the features extracted for each distance and orientation value from each local region then concatenate the features extracted from each local region to 
obtain the holistic description of the face. Calculating the Haralick features is handled via the Haralick function, an mfile found on Matlab's File Exchange Website written by Stefan Winzeck in 2012. The computations of the features in Haralick's original paper are based on the calculations found in Miyamoto's paper [21] for fast calculations.

2) GLRLM features : [6][7][8] derive descriptors from a run-length matrix that is based on characteristics of the gray level runs within a given image. A gray level run is a set of consecutive pixels with the same value, and the run length is the number of pixels in the set. The run-length matrix $P$ contains in each location $p(i, j)$ the number of runs of length $\mathrm{j}$ at a given gray level $\mathrm{i}$. In this study, The two features (Run Length Nonuniformity (RLN), Gray Level Nonuniformity (GLN)) are selected and calculated from each GLRLM evaluated at four orientations $\mathrm{h}=\left\{0^{\circ}, 45^{\circ}, 90^{\circ}\right.$, $135^{\circ}$ \} after quantizing to 8 gray levels. A descriptor is obtained by concatenating the features extracted for each orientation value from each local region. Then, concatenate the features extracted from each local region to obtain the holistic description of the face.

D. Classification : we train linear SVM [22] using LibSVM to determine the person's identity. The evaluation criterion is the accuracy of the recognition system (\%).

\section{RESULTS AND DISCUSSION:}

First, we do many experiments to select distance parameter values, the number of local regions, the subset of Haralick features. Then, depending on the selected values we study the effect of adding pre-processing step to the system after comparing different pre-processing methods and select the best. Finally, evaluate the proposed system on another type of features.

\section{A. Select the parameters values:}

1) Distance parameter: divide ORL images to $2 * 2$ local regions, for each region 4-GLCMs are builded (8 quantization levels) according to the four orientations. Then, from each GLCM the 13-Haralick features are extracted and without pre-processing step. The obtained results are shown in (table I). we select $\{1,3,5,7\}$ because it gives the best result.

TABLE I. EFFECT OF DISTANCE PARAMETER VALUES ON ACCURACY

\begin{tabular}{|c|c|c|c|c|c|c|c|c|c|c|c|}
\hline \multirow{2}{*}{ distance } & \multicolumn{10}{|c|}{ Random sets } \\
\cline { 2 - 12 } & $\boldsymbol{R} 1$ & $\boldsymbol{R} 2$ & $\boldsymbol{R 3}$ & $\boldsymbol{R 4}$ & $\boldsymbol{R 5}$ & $\boldsymbol{R 6}$ & $\boldsymbol{R 7}$ & $\boldsymbol{R} \boldsymbol{R}$ & $\boldsymbol{R} \boldsymbol{R}$ & $\boldsymbol{R} 10$ & $\boldsymbol{a v g}$ \\
\hline $\mathbf{1}$ & 61.6 & 65.8 & 59.1 & 55 & 53.3 & 63.3 & 63.3 & 59.1 & 68.3 & 62.5 & $\mathbf{6 1 . 1 3}$ \\
\hline $\mathbf{1 , 3}$ & 62.5 & 64.1 & 60.8 & 55.8 & 56.6 & 68.3 & 69.1 & 60.8 & 70.8 & 68.3 & $\mathbf{6 3 . 7 1}$ \\
\hline $\mathbf{1 , 3 , 5 , 7}$ & 70.8 & 68.3 & 70.8 & 62.5 & 65 & 71.6 & 72.5 & 70.8 & 75 & 75.8 & $\mathbf{7 0 . 3 1}$ \\
\hline
\end{tabular}

2) The number of local regions: according to the results shown in (table II,table III), dividing ORL images to $3 * 3$ and Yale images to $3 * 4$ improved the results. Nevertheless, increasing the number of local regions will improve the result only to a certain extent.
TABLE II. EFFECT OF LOCAL REGIONS ON ACCURACY (ORL)

\begin{tabular}{|c|c|c|c|c|c|c|c|c|c|c|c|}
\hline \multirow{2}{*}{ \#local regions } & \multicolumn{7}{|c|}{ Random sets } \\
\cline { 2 - 11 } & $\boldsymbol{R} \mathbf{1}$ & $\boldsymbol{R 2}$ & $\boldsymbol{R 3}$ & $\boldsymbol{R 4}$ & $\boldsymbol{R 5}$ & $\boldsymbol{R 6}$ & $\boldsymbol{R 7}$ & $\boldsymbol{R} 8$ & $\boldsymbol{R} 9$ & $\boldsymbol{R} 10$ & avg \\
\hline $\mathbf{2} * \mathbf{2}$ & 70.8 & 68.3 & 70.8 & 62.5 & 65 & 71.6 & 72.5 & 70.8 & 75 & 75.8 & $\mathbf{7 0 . 3}$ \\
\hline $\mathbf{3} * \mathbf{3}$ & 80 & 81.6 & 87.5 & 80.8 & 76.6 & 80.8 & 84.16 & 87.5 & 83.3 & 83.3 & $\mathbf{8 2 . 5}$ \\
\hline
\end{tabular}

TABLE III. EFFECT OF LOCAL REGIONS ON ACCURACY (YALE)

\begin{tabular}{|c|c|c|c|c|c|c|c|c|c|c|c|}
\hline \multirow{2}{*}{ \#local regions } & \multicolumn{7}{|c|}{ Random sets } \\
\cline { 2 - 12 } & $\boldsymbol{R 1}$ & $\boldsymbol{R 2}$ & $\boldsymbol{R 3}$ & $\boldsymbol{R 4}$ & $\boldsymbol{R 5}$ & $\boldsymbol{R 6}$ & $\boldsymbol{R 7}$ & $\boldsymbol{R} 8$ & $\boldsymbol{R 9}$ & $\boldsymbol{R} 10$ & $\boldsymbol{a v g}$ \\
\hline $\mathbf{2 * 2}$ & 48.8 & 46.6 & 37.7 & 53.3 & 57.7 & 51.1 & 46.6 & 33.3 & 60 & 62.2 & $\mathbf{4 9 . 7}$ \\
\hline $\mathbf{3} * \mathbf{4}$ & 63.7 & 64.1 & 60.8 & 55.8 & 56.6 & 68.3 & 69.1 & 60.8 & 70.8 & 68.3 & $\mathbf{6 2 . 5}$ \\
\hline
\end{tabular}

3) select the subset of Haralick features:

In this study, we select the five features (difference variance, difference entropy, sum entropy, two information measures of correlation) from a set of 13 Haralick descriptors is calculated from each co-occurrence matrix evaluated at four orientations $\mathrm{h}=\left\{0^{\circ}, 45^{\circ}, 90^{\circ}, 135^{\circ}\right\}$ and with distance $d=\{1,3,5,7\}$. The results shown in (table IV, table $\mathrm{V}$ ) indicate that the less correlated features improved the accuracy significantly.

TABLE IV. EFFECT OF SELECTING FEATURES ON ACCURACY (ORL)

\begin{tabular}{|c|c|c|c|c|c|c|c|c|c|c|c|}
\hline \multirow{2}{*}{ features } & \multicolumn{10}{|c|}{ Random sets } \\
\cline { 2 - 11 } & $\boldsymbol{R} \boldsymbol{1}$ & $\boldsymbol{R 2}$ & $\boldsymbol{R 3}$ & $\boldsymbol{R 4}$ & $\boldsymbol{R 5}$ & $\boldsymbol{R 6}$ & $\boldsymbol{R 7}$ & $\boldsymbol{R} \boldsymbol{R}$ & $\boldsymbol{R} 9$ & $\boldsymbol{R} 10$ & $\boldsymbol{a v g}$ \\
\hline 13-haralick & 80 & 81.6 & 87.5 & 80.8 & 76.6 & 80.8 & 84.1 & 87.5 & 83.3 & 83.3 & $\mathbf{8 2 . 5}$ \\
\hline 5-selected & 97.5 & 96.9 & 97.5 & 99.1 & 96.6 & 96.6 & 99.1 & 97.5 & 99.1 & 98.3 & $\mathbf{9 7 . 8}$ \\
\hline
\end{tabular}

TABLE V. EFFECT SELECTING FEATURES ON ACCURACY (YALE)

\begin{tabular}{|c|c|c|c|c|c|c|c|c|c|c|c|}
\hline \multirow{2}{*}{ features } & \multicolumn{10}{|c|}{ Random sets } \\
\cline { 2 - 12 } & $\boldsymbol{R 1}$ & $\boldsymbol{R 2}$ & $\boldsymbol{R 3}$ & $\boldsymbol{R 4}$ & $\boldsymbol{R 5}$ & $\boldsymbol{R 6}$ & $\boldsymbol{R 7}$ & $\boldsymbol{R} 8$ & $\boldsymbol{R} 9$ & $\boldsymbol{R 1 0}$ & $\boldsymbol{a v g}$ \\
\hline 13-haralick & 62.2 & 64.4 & 57.7 & 55.5 & 51.1 & 55.5 & 68.8 & 55.5 & 71.1 & 68.8 & $\mathbf{6 1}$ \\
\hline 5 selected & 82.2 & 84.4 & 84.4 & 86.6 & 73.3 & 84.4 & 80 & 80 & 86.6 & 84.4 & $\mathbf{8 2 . 6}$ \\
\hline
\end{tabular}

We evaluated the proposed descriptor on ORL dataset by comparing it with some existing local feature descriptors. In this experiment, all of these features were implemented on the same scenario for different number of training images per person. The effect of applying each of these descriptors were tested and compared with proposed features on ORL dataset. The obtained results are shown in (table VI).

TABLE VI. THE COMPARISON OF THE PROPOSED DESCRIPTOR WITH OTHER EXISTING DESCRIPTORS

\begin{tabular}{|c|c|c|c|}
\hline \multirow{2}{*}{ features } & \multicolumn{3}{|c|}{ Avg of 10 Random sets } \\
\cline { 2 - 4 } & $\mathbf{5}$ & $\mathbf{7}$ & $\mathbf{8}$ \\
\hline Uniform LBP & 73.6 & 80.8 & 82.6 \\
\hline $\begin{array}{c}\text { Multi-block } \\
\text { uniform LBP }\end{array}$ & 91.45 & 95.2 & 96.5 \\
\hline Proposed features & 95.5 & 97.8 & 97.62 \\
\hline
\end{tabular}

\section{B. Effect of pre-processing step:}

This step enhance the results on ORL images and contribute to lighting compensation for YALE images as 
shown in (table VII, table VIII). We select SQI for lighting compensation depending on results shown in (table IX).

TABLE VII. EFFECT PRE-PROCESSING STEP ON ACCURACY (ORL)

\begin{tabular}{|c|c|c|c|c|c|c|c|c|c|c|c|}
\hline \multirow{2}{*}{ method } & \multicolumn{11}{|c|}{ Random sets } \\
\hline & $R 1$ & $R 2$ & $R 3$ & $R 4$ & $R 5$ & $R 6$ & $R 7$ & $R 8$ & $R 9$ & $R 10$ & avg \\
\hline $\begin{array}{c}\text { No pre- } \\
\text { processing }\end{array}$ & 97.5 & 96.9 & 97.5 & 99.1 & 96.6 & 96.6 & 99.1 & 97.5 & 99.1 & 98.3 & 97.8 \\
\hline GC & 99.1 & 99.1 & 100 & 98.3 & 97.5 & 99.15 & 99.1 & 100 & 99.1 & 100 & 99.1 \\
\hline
\end{tabular}

TABLE VIII. EFFECT PRE-PROCESSING STEP ON ACCURACY (YALE)

\begin{tabular}{|c|c|c|c|c|c|c|c|c|c|c|c|}
\hline \multirow{2}{*}{ method } & \multicolumn{10}{|c|}{ Random sets } \\
\cline { 2 - 12 } & $\boldsymbol{R 1}$ & $\boldsymbol{R 2}$ & $\boldsymbol{R 3}$ & $\boldsymbol{R 4}$ & $\boldsymbol{R 5}$ & $\boldsymbol{R 6}$ & $\boldsymbol{R} 7$ & $\boldsymbol{R} \boldsymbol{8}$ & $\boldsymbol{R} \boldsymbol{9}$ & $\boldsymbol{R 1 0}$ & $\boldsymbol{a v g}$ \\
\hline $\begin{array}{c}\text { No pre- } \\
\text { processing }\end{array}$ & 82.2 & 84.4 & 84.4 & 86.6 & 73.3 & 84.4 & 80 & 80 & 86.6 & 84.4 & $\mathbf{8 2 . 6}$ \\
\hline SQI & 95.5 & 93.3 & 93.3 & 97.7 & 93.3 & 88.8 & 93.3 & 93.3 & 95.5 & 95.5 & $\mathbf{9 4}$ \\
\hline
\end{tabular}

TABLE IX. COMPARING DIFFERENT PRE-PROCESSING METHODES [12](YALE)

\begin{tabular}{|c|c|c|c|c|c|c|c|c|c|c|c|}
\hline \multirow{2}{*}{ method } & \multicolumn{10}{|c|}{ Random sets } \\
\cline { 2 - 12 } & $\boldsymbol{R} \boldsymbol{1}$ & $\boldsymbol{R} \boldsymbol{2}$ & $\boldsymbol{R 3}$ & $\boldsymbol{R 4}$ & $\boldsymbol{R 5}$ & $\boldsymbol{R 6}$ & $\boldsymbol{R} 7$ & $\boldsymbol{R} \boldsymbol{8}$ & $\boldsymbol{R} \boldsymbol{9}$ & $\boldsymbol{R} \mathbf{1 0}$ & $\boldsymbol{a v g}$ \\
\hline SQI & 95.5 & 93.3 & 93.3 & 97.7 & 93.3 & 88.8 & 93.3 & 93.3 & 95.5 & 95.5 & $\mathbf{9 4}$ \\
\hline LDCT & 93.3 & 88.8 & 84.4 & 93.3 & 80 & 88.8 & 93.3 & 91.1 & 93.3 & 86.6 & $\mathbf{8 9 . 3}$ \\
\hline TT & 97.7 & 91.1 & 91.1 & 97.7 & 84.4 & 86.6 & 93.3 & 88.8 & 88.8 & 86.6 & $\mathbf{9 0}$ \\
\hline LT & 86.6 & 86.6 & 86.6 & 91.1 & 84.4 & 88.8 & 93.3 & 86.6 & 95.5 & 93.3 & $\mathbf{8 9 . 3}$ \\
\hline
\end{tabular}

We also compared the proposed method in equal conditions with other state-of-the-art methods on the ORL dataset. In this comparison only 5 images were used as reference images for training and the remained 5 were used for test. Table $\mathrm{X}$ shows the results obtained from the comparison of the proposed method and state-of-the-art methods.

TABLE X. The RESUlt OF APPLYING THE PROPOSED METHOD AND THE OTHER EXISTING METHOD ON ORL DATABASE

\begin{tabular}{|c|c|}
\hline Approach & Accuracy \\
\hline Haralick features[23] & 79.1 \\
\hline Direct GLCM[23] & 91.6 \\
\hline Proposed method & 92.5 \\
\hline
\end{tabular}

\section{GLRLM features:}

First, study the effect of selecting GLRLM features (GLN, RLN) on accuracy, the results are shown in (table $\mathrm{XI})$ indicate that the selected features improved the accuracy significantly and reduce the feature vector dimensions. Then, study the effect of pre-processing on selected features. The results are shown in (table XII) indicate that the pre-processing step enhances the accuracy on ORL and improves the accuracy significantly on YALE.
TABLE XI. COMPARING DIFFERENT GLRLM FEATURES (ORL, YALE) WITH PRE-PROCESSING

\begin{tabular}{|c|c|c|c|c|c|c|}
\hline \multirow{3}{*}{$\begin{array}{l}\text { Random } \\
\text { sets }\end{array}$} & \multicolumn{6}{|c|}{ features } \\
\hline & \multicolumn{3}{|c|}{ ORL } & \multicolumn{3}{|c|}{ YALE } \\
\hline & $\begin{array}{c}\text { Galloway(2015) } \\
{[6]+\text { Chu et }} \\
\text { al.(1990) [7] }\end{array}$ & \begin{tabular}{|c} 
Holder \\
et \\
al.(1990) \\
{$[8]$}
\end{tabular} & $\begin{array}{l}\text { Selected } \\
\text { features }\end{array}$ & $\begin{array}{c}\text { Galloway(2015) } \\
{[6]+\text { Chu et }} \\
\text { al.(1990) [7] }\end{array}$ & $\begin{array}{c}\text { Holder } \\
\text { et } \\
\text { al.(1990) } \\
{[8]}\end{array}$ & \begin{tabular}{|l} 
Selected \\
features
\end{tabular} \\
\hline $\mathbf{R 1}$ & 91.6 & 87.5 & 98.3 & 80 & 80 & 91.1 \\
\hline $\mathbf{R 2}$ & 90 & 84.1 & 100 & 75.5 & 71.1 & 86.6 \\
\hline $\mathbf{R 3}$ & 92.5 & 85.8 & 97.5 & 82.2 & 73.3 & 88.8 \\
\hline R4 & 85.8 & 85 & 94.1 & 73.3 & 75.5 & 91.1 \\
\hline R5 & 88.3 & 87.5 & 100 & 77.7 & 73.3 & 88.8 \\
\hline R6 & 92.5 & 85.8 & 96.6 & 75.5 & 82.2 & 88.8 \\
\hline R7 & 94.16 & 93.3 & 99.1 & 84.4 & 80 & 93.3 \\
\hline R8 & 92.5 & 85.8 & 97.5 & 71.1 & 71.1 & 91.1 \\
\hline R9 & 93.3 & 89.1 & 98.3 & 80 & 77.7 & 93.3 \\
\hline R10 & 92.5 & 90.8 & 98.3 & 86.6 & 82.2 & 93.3 \\
\hline avg & 91.3 & 87.5 & 98 & 78.6 & 76.6 & 90.6 \\
\hline
\end{tabular}

TABLE XII. EFFECT OF PRE-PROCESSING ON SELECTED GLRLM FEATURES (ORL, YALE)

\begin{tabular}{|c|c|c|c|c|}
\hline \multirow{2}{*}{$\begin{array}{c}\text { Random } \\
\text { sets }\end{array}$} & \multicolumn{4}{|c|}{ Pre-processing methods } \\
\cline { 2 - 5 } & $\begin{array}{c}\text { WRL } \\
\text { processing }\end{array}$ & \multicolumn{1}{c|}{ GC } & $\begin{array}{c}\text { Without pre- } \\
\text { processing }\end{array}$ & \multicolumn{1}{c|}{ SQI } \\
\hline \multirow{2}{*}{ R1 } & 98.3 & 98.3 & 77.7 & 91.1 \\
\hline R2 & 96.6 & 100 & 68.8 & 86.6 \\
\hline R3 & 96.6 & 97.5 & 71.1 & 88.8 \\
\hline R4 & 95 & 94.1 & 77.7 & 91.1 \\
\hline R5 & 95 & 100 & 64.4 & 88.8 \\
\hline R6 & 97.5 & 96.6 & 80 & 88.8 \\
\hline R7 & 98.3 & 99.1 & 75.5 & 93.3 \\
\hline R8 & 96.6 & 97.5 & 73.3 & 91.1 \\
\hline R9 & 96.6 & 98.3 & 82.2 & 93.3 \\
\hline R10 & 94.1 & 98.3 & 82.2 & 93.3 \\
\hline avg & $\mathbf{9 6 . 5}$ & $\mathbf{9 8}$ & $\mathbf{7 5 . 3}$ & $\mathbf{9 0 . 6}$ \\
\hline
\end{tabular}

\section{CONCLUSION}

We show in this paper that it is possible to improve the performance of different descriptors extracted from the cooccurrence matrix and gray-level run length matrix by coupling them with local approach. Using smaller number of gray levels made the algorithm faster and, at the same time, preserved the high recognition rate. Our main intention, is to select small set of features which are less correlated, compare different pre-processing methods applied before the matrix extraction to find the best and do many experiments to choose appropriate values for different parameters. 
Future experiments, evaluate this system on another databases in the wild, test it for different problems in recognition. Search for a deep learning model to extract features and compare the performance between deep features and handcrafted features.

\section{REFERENCES}

[1] W. Zhao, R. Chellappa, P. J. Phillips, and A. Rosenfeld, "Face recognition: A literature survey," ACM Comput. Surv., vol. 35, no. 4, pp. 399-458, 2003.

[2] M. Wang and W. Deng, "Deep face recognition: a survey," arXiv Prepr. arXiv1804.06655, 2018.

[3] T. Ahonen, A. Hadid, and M. Pietikainen, "Face description with local binary patterns: Application to face recognition," IEEE Trans. Pattern Anal. Mach. Intell., vol. 28, no. 12, pp. 2037-2041, 2006.

[4] M. A. Ochoa-Villegas, J. A. Nolazco-Flores, O. BarronCano, and I. A. Kakadiaris, "Addressing the illumination challenge in two-dimensional face recognition: a survey," IET Comput. Vis., vol. 9, no. 6, pp. 978-992, 2015.

[5] R. M. Haralick, K. Shanmugam, and I. H. Dinstein, "Textural features for image classification," IEEE Trans. Syst. Man. Cybern., no. 6, pp. 610-621, 1973.

[6] M. M. Galloway, "Texture classification using gray level run length," Comput. Graph. image Process., vol. 4, no. 2, pp. $172-179,1975$.

[7] A. Chu, C. M. Sehgal, and J. F. Greenleaf, "Use of gray value distribution of run lengths for texture analysis," Pattern Recognit. Lett., vol. 11, no. 6, pp. 415-419, 1990.

[8] B. V Dasarathy and E. B. Holder, "Image characterizations based on joint gray level-run length distributions," Pattern Recognit. Lett., vol. 12, no. 8, pp. 497-502, 1991.

[9] A. Eleyan and H. Demirel, "Co-occurrence based statistical approach for face recognition," in 2009 24th international symposium on computer and information sciences, 2009, pp. 611-615.

[10] S. Ribaric and M. Lopar, "Palmprint recognition based on local Haralick features," in 2012 16th IEEE

Mediterranean Electrotechnical Conference, 2012, pp. 657-660.

[11] X. Tan and W. Triggs, "Enhanced local texture feature sets for face recognition under difficult lighting conditions," IEEE Trans. image Process., vol. 19, no. 6, pp. 1635-1650, 2010.
[12] H. Han, S. Shan, X. Chen, and W. Gao, "A comparative study on illumination preprocessing in face recognition," Pattern Recognit., vol. 46, no. 6, pp. 1691-1699, 2013.

[13] L. Nanni, S. Brahnam, S. Ghidoni, and E. Menegatti, "Improving the descriptors extracted from the cooccurrence matrix using preprocessing approaches," Expert Syst. Appl., vol. 42, no. 22, pp. 8989-9000, 2015.

[14] S. Zafeiriou, C. Zhang, and Z. Zhang, "A survey on face detection in the wild: past, present and future," Comput. Vis. Image Underst., vol. 138, pp. 1-24, 2015.

[15] P. Viola and M. Jones, "Rapid object detection using a boosted cascade of simple features," in Proceedings of the 2001 IEEE computer society conference on computer vision and pattern recognition. CVPR 2001, 2001, vol. 1, pp. I-I.

[16] E. Alionte and C. Lazar, "A practical implementation of face detection by using Matlab cascade object detector," in 2015 19th International Conference on System Theory, Control and Computing (ICSTCC), 2015, pp. 785-790.

[17] Y. Li, C. Wang, and X. Ao, Illumination processing in face recognition. InTech, 2010.

[18] A. Shashua and T. Riklin-Raviv, "The quotient image: Class-based re-rendering and recognition with varying illuminations," IEEE Trans. Pattern Anal. Mach. Intell., vol. 23, no. 2, pp. 129-139, 2001.

[19] H. Wang, S. Z. Li, and Y. Wang, "Generalized quotient image," in Proceedings / CVPR, IEEE Computer Society Conference on Computer Vision and Pattern Recognition, 2004, pp. 498-505.

[20] X. Tan and B. Triggs, "Preprocessing and feature sets for robust face recognition," in Proceedings of the IEEE Conference on Computer Vision and Pattern Recognition (CVPR'07), 2007, vol. 7, pp. 1-8.

[21] E. Miyamoto and T. Merryman, "Fast calculation of Haralick texture features," Hum. Comput. Interact. institute, Carnegie Mellon Univ. Pittsburgh, USA. Japanese Restaur. Off., 2005.

[22] B. E. Boser, I. M. Guyon, and V. N. Vapnik, "A training algorithm for optimal margin classifiers," in Proceedings of the fifth annual workshop on Computational learning theory, 1992, pp. 144-152.

[23] A. Eleyan and H. Demirel, "Co-occurrence matrix and its statistical features as a new approach for face recognition," Turkish J. Electr. Eng. Comput. Sci., vol. 19, no. 1, pp. 97-107, 2011. 